\title{
Too good to be true
}

Many a scientist has been seduced by an elegant idea, only to find that aesthetics are not always a good guide to a theory's accuracy. Of all the beautiful theories slain by ugly facts, which most deserved to be right?

\section{John Maynard Smith}

W ehave all had the experience of having what seemed like a great idea, only to find that it wouldn't do, because it was contradicted by a familiar fact or suffered from an incurable logical error. The experience prompts the question: what was the cleverest idea in the history of science that turned out to be wrong?

Before you try to answer this question, let me insist that by 'wrong' I mean really wrong. I won't accept 'Newton's laws of motion' as an answer. I understand that physicists nowadays insist that his laws break down at very high velocities and accelerations. As an ex-engineer whose aeroplanes never flew faster than sound, let alone light, I have great faith in Newton's laws: they are not so much 'wrong' as true subject to certain constraints. For the same reason, I would not accept 'Mendel's laws' as an answer either, even though the law of independent assortment is false for genes on the same chromosome. I want an idea that is wrong in all circumstances, but which deserves to be right.

I would not be asking this question if I did not have a candidate answer. In the physical sciences, one could suggest Kelvin's estimate of the age of the Earth, based on the expected rate of cooling, but wrong because Kelvin knew nothing of radioactive heating. The idea was wrong, and important, but perhaps not terribly clever. A better candidate, I think, would be the steady-state theory of the Universe. I find it a much more attractive notion than the Big Bang, but I have to take it on trust that it is mistaken.

My favoured candidate is from biology. In 1957, Francis Crick, John Griffith and Leslie Orgel published a paper explaining why only 20 amino acids are used in proteins, although other possible amino acids exist (Proc. Natl Acad. Sci.USA 43, 416-421; 1957). It was already known that the sequence of amino acids in a protein was determined by the sequence of four kinds of base - A, C, G and $\mathrm{T}$ - in DNA, but the 'code' connecting the base sequence to the amino-acid sequence was unknown. It was widely assumed that this would be a triplet code. It could not be a doublet code, as at best this could specify only 16 amino acids. The idea that it resembled Morse code, with different numbers of bases specifying different amino acids, although possible, was not thought likely.

The authors argued as follows. Granted that it is a triplet code, how does the cell know which triplets to read? There are no 'spaces' in DNA to separate them, analogous to the

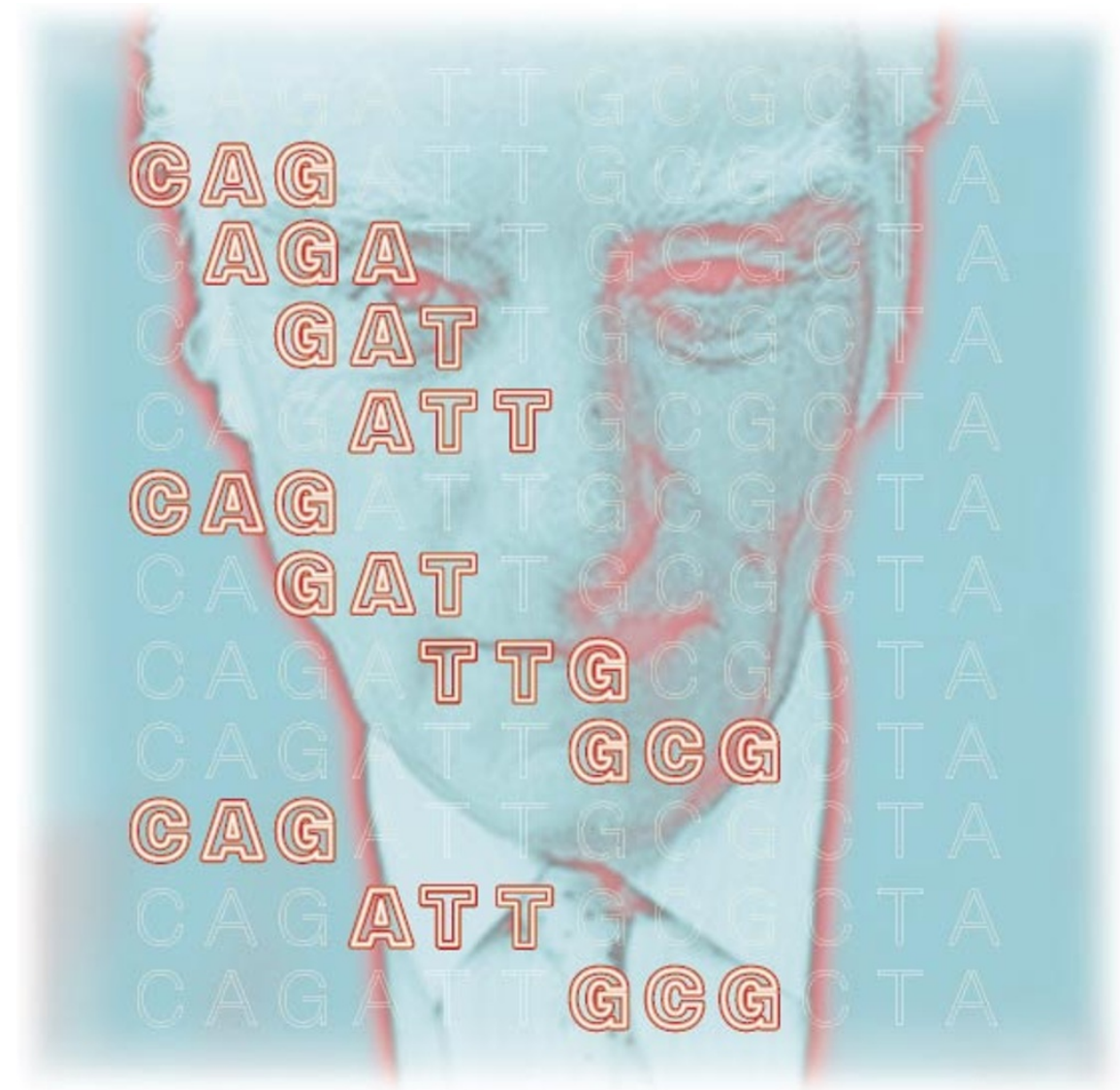

No matter in what order these meaningful triplets are arranged, none of the 'out of frame' triplets must be meaningful.

spaces that separate letters in Morse code. It is implausible to suppose that translation starts at the beginning of a gene and counts off in threes. Instead, they suggested that only some of the triplets are meaningful, in the sense of specifying an amino acid. No matter in what order these meaningful triplets are arranged, none of the 'out of frame' triplets must be meaningful. For example, if ACT and GCA are meaningful, then CTA, TAC, AGC, CAG, CTG, TGC, CAA and AAC must not be. Then a message can be read in only one way. If that is how it works, what is the largest number of meaningful triplets that is possible? The answer, of course, is 20 .

The authors treated their idea with due caution, writing: "We present the solution here because it gives the 'magic number' 20 , so that our answer may perhaps be of biological significance". If it had been my idea, I would be believing it still. But we now know that 61 of the 64 triplets specify an amino acid. The cell does indeed start at the beginning of a gene and count off in threes. If one or two nucleotides are deleted, then every amino acid beyond the deletion is wrong, and the protein is useless. If three are deleted, translation gets back into frame, and all is not lost.

It is a remarkable twist in the history of science that Crick (pictured) was one of the authors of the paper that used this argument to show that the code is indeed a triplet code, and that the cell does count in threes (Crick, F. H. C., Barnett, L., Brenner, S. \& WattsTobin, R. J. Nature 192, 1227-1232; 1961).

Since writing the above, I find I am not alone in my choice. Twenty years ago (what is it about 20?), describing the above argument, Horace Freeland Judson wrote: "This was the beginning of the comma-free code - an idea of Crick's that was the most elegant biological theory ever to be proposed and proved wrong" (The Eighth Day of Creation, Cape, 1979).

John Maynard Smith is at the School of Biological

Sciences, University of Sussex, Falmer, Brighton BN1 9QG, UK. 